\title{
A EMPATIA E A PERCEPÇÃO DE EMOÇõES EM ESTUDANTES DE PSICOLOGIA E PSICOTERAPEUTAS
}

\author{
Ana Rita Palhoco \\ Mestre, Faculdade de Psicologia da Universidade de Lisboa. \\ Maria João Afonso \\ Doutora, Faculdade de Psicologia da Universidade de Lisboa.
}

\begin{abstract}
Resumo
A presente investigação teve como objectivo estudar a empatia e a capacidade de percepção de emoções primárias em estudantes de Psicologia e psicoterapeutas da Faculdade de Psicologia da Universidade de Lisboa. Para medir os construtos em estudo, recorreu-se à primeira versão experimental portuguesa do Test de Empatía Cognitiva y Afectiva (TECA), traduzida e adaptada na presente investigação, e ao Teste de Percepção de Emoções Primárias (PEP), construído na Universidade de São Francisco (Brasil). Procurou-se averiguar em que medida as competências supramencionadas são desenvolvidas na formação académica ou apenas através da experiência prática, pelo que se recorreu à sua avaliação, numa amostra de 113 participantes. Verificou-se que a empatia cognitiva é superior nos sujeitos com maior formação e experiência, que a empatia emocional está positivamente (porém não significativamente) correlacionada com a percepção de emoções, e que os dois tipos de empatia (cognitiva e emocional) apresentam algum grau de independência entre si.

Palavras-chave: empatia, percepção de emoções primárias, psicoterapia.
\end{abstract}

\section{EMPATHY AND EMOTIONAL PERCEPTION IN PSYCHOLOGY STUDENTS AND PSYCHOTHERAPISTS}

\begin{abstract}
This research project aimed the study of the empathic ability and the perception of primary emotions, both in students and psychotherapists at the Faculty of Psychology, University of Lisbon. To assess these variables two tests were used: the first Portuguese experimental version of the Test de Empatía Cognitiva y Afectiva (TECA), translated and adapted as part of this study, and the Teste de Percepção de Emoções Primárias (PEP), developed at the University of San Francisco (Brazil). The intent was to ascertain if these abilities may be developed through academic teaching or only by practice, whereby it was assessed in a sample of 113 participants. It was found that cognitive empathy is higher in individuals with better knowledge and experience, that emotional empathy is positively (but not significantly) correlated with the perception of emotions and that there is evidence of a certain degree of independence between the two types of empathy (cognitive and emotional).

Keywords: empathy, perception of primary emotions, psychotherapy.
\end{abstract}




\title{
LA EMPATÍA Y LA PERCEPCIÓN DE EMOCIONES EN ESTUDIANTES DE PSICOLOGIA Y PSICOTERAPEUTAS
}

\begin{abstract}
Resumen
La presente investigación tuvo el objetivo de estudiar la empatía e la capacidad de reconocer emociones primarias, en estudiantes de psicología y en psicoterapeutas, de la Facultad de Psicología de la Universidad de Lisboa. Para medir los constructos en estudio se ha utilizado la primera versión experimental portuguesa del Test de Empatía Cognitiva y Afectiva (TECA), traducida y adaptada en la presente investigación, y el Teste de Percepção de Emoções Primárias (PEP), construido en la Universidad de San Francisco (Brasil). Se intentó averiguar si las competencias referidas anteriormente se desarrollan con la formación académica o solamente con la experiencia práctica, y para eso se recorrió à su evaluación, en una muestra de 113 participantes. Se ha encontrado que la empatía cognitiva es superior en los sujetos que tienen mayor formación y experiencia, que la empatía emocional es positivamente (pero no significativamente) correlacionada con la percepción de emociones, y que existe algún grado de independencia entre los dos tipos de empatía (cognitiva y emocional).
\end{abstract}

Palabras clave: empatía, percepción de emociones primarias, psicoterapia.

\section{INTRODUÇÃO}

A Psicologia Clínica encontra-se actualmente numa era de convergência, direccionada para conceitos transversais integradores de perspectivas, emergindo desta forma um factor comum, imprescindível a qualquer processo terapêutico: a relação entre terapeuta e cliente. Esta relação, também denominada de aliança terapêutica, comporta vários componentes: 1) acordo entre o terapeuta e o cliente acerca das tarefas terapêuticas; 2) concordância entre ambos acerca dos objectivos da terapia; e 3) estabelecimento de um laço terapêutico de qualidade, com empatia e congruência, que facilita a confiança e aceitação entre o terapeuta e o cliente (Bordin, 1979). A empatia, a genuinidade e a visão positiva incondicional constituem, por sua vez, qualidades essenciais que um terapeuta deverá idealmente apresentar (Rogers, 1974). No presente estudo, apenas será dado relevo ao conceito de empatia, que é analisado segundo uma perspectiva bidimensional, com distinção entre a empatia cognitiva e a emocional, e compreendido como uma característica dos terapeutas, eficaz no estabelecimento de uma comunicação emocional com o cliente e que o ajuda a reflectir sobre as suas emoções (Greenberg, 2009).

Esta integração de perspectivas encontra paralelo na concepção de Inteligência Emocional, a qual se baseia numa síntese da componente intelectual e com o processamento emocional, sendo definida como a capacidade de percepcionar adequadamente, avaliar e expressar emoções, assim como de recorrer ao pensamento com os propósitos de as compreender e de dominar o 
seu conhecimento, o que viabiliza a própria regulação e o controlo emocional (Mayer, Salovey \& Caruso, 2004). A ligação estabelecida provém do facto de este conceito englobar não só duas componentes, cognitiva e emocional, mas também a sua aplicação em dois níveis distintos, o inter- e o intra-pessoal, na medida em que visa, entre outros aspectos, a identificação e compreensão de emoções, em si mesmo e no outro.

Nesta investigação, o interesse teórico recai particularmente sobre o nível inter-pessoal, relativamente à relação entre terapeuta e cliente, considerando como competências integrantes deste construto a percepção adequada de emoções e a subsequente avaliação das mesmas, que implica o reconhecimento de expressões faciais e posturais, assim como a capacidade de compreensão emocional, isto é, de designação das emoções observadas (Mayer et al., 2004).

Numa análise aprofundada dos construtos em estudo, importa salientar que a aliança terapêutica, quando estudada por Norcross (2002, citado por Gilbert, 2009), apresentou dados sólidos que comprovam a sua relevância ao nível do sucesso terapêutico, na medida em que 15\% desse sucesso advém do efeito que as expectativas do cliente têm na mudança, 15\% está associado às técnicas utilizadas (específicas de cada orientação teórica), 30\% deve-se aos "factores comuns", de onde se destaca a aliança terapêutica, e os restantes $40 \%$ correspondem à mudança extra-terapêutica.

Neste âmbito, a empatia, componente da aliança terapêutica em estudo, diz respeito à capacidade de compreender o outro através do seu ponto de referência (Cormier, Nurius \& Osborn, 2009). Contudo, para que um terapeuta seja empático, não se pode limitar a reflectir sobre os pensamentos do cliente, tendo de ser capaz de ressoar emocionalmente a experiência deste (Gilbert \& Leahy, 2009), de modo a que ele desenvolva um sentimento de aceitação pessoal e vivencie uma "experiência emocional correctiva" (Kohut, 1984, citado por Cormier et al., 2009), isto é, compreenda que as suas emoções são válidas e fazem sentido (Bohart \& Greenberg, 1997).

Como esta capacidade exige que o terapeuta consiga ressoar a experiência do cliente, isto implica que este apresente responsividade terapêutica, isto é, que adeqúe o conteúdo e a forma da comunicação ao longo da sessão, de acordo com o impacto que esta apresenta no cliente (Vasco, 2007). No entanto, para que isto ocorra, o terapeuta necessita de estar em sintonia empática com os sentimentos do paciente e com os seus significados associados, pois só assim 
poderá perceber quais os objectivos deste e encontrar colaborativa e progressivamente as tarefas que permitirão atingi-los (Greenberg, 2009).

Apesar de a empatia ser definida por alguns autores numa perspectiva mais emocional (e.g. Batson; Hoffman; Lipps; Mehrabian \& Epstein; Stotland), e por outros num âmbito mais cognitivo (e.g. Dymond; Hogan; Köhler; Mead; Salovey \& Mayer), a existência desta concomitante sintonia e compreensão emocionais levou alguns autores a adoptarem uma perspectiva integradora (e.g. Davis, Eisenberg, Hoffman) tendo em consideração os seus componentes cognitivos e emocionais (Fernández-Pinto, López-Pérez \& Márquez, 2008). Esta perspectiva bidimensional (empatia cognitiva e emocional) é aplicada ao contexto terapêutico por Gladstein (1983), que chama a atenção para a necessidade de distinguir que a empatia cognitiva consiste numa percepção da realidade do cliente a partir do seu ponto de vista, e que a empatia emocional diz respeito à capacidade de sentir com ele, mantendo a distância emocional necessária para não perder a objectividade.

Este estabelecimento e manutenção das relações compreendem a demonstração dos estados emocionais de modo verbal e não verbal, o que permite ao terapeuta adequar o seu comportamento, momento a momento, em função do que vai percepcionando. Assim, para que um terapeuta possa estabelecer uma relação empática com o cliente, necessita de percepcionar as emoções que este expressa, para posteriormente devolver a informação recebida e pensar colaborativamente com ele, demonstrando envolvimento na comunicação e compreensão empática.

Neste âmbito, sabe-se que os terapeutas experientes apresentam maior semelhança na prática clínica com terapeutas experientes de outras orientações, do que com terapeutas inexperientes da mesma orientação teórica (Fiedler, 1950, citado por Vasco, 2005), e verifica-se frequentemente que os terapeutas principiantes não possuem nem muitos recursos atencionais ao nível das capacidades interpessoais, nem muitas competências técnicas, pelo que tendem a favorecer as últimas (Bennett-Levy \& Thwaites, 2009). É assim legítimo depreender que os terapeutas inexperientes, embora não possuam conhecimentos técnicos muito aprofundados, apreenderam previamente a aplicabilidade e adequação das técnicas em função da idiossincrasia dos clientes, o que Ihes é proporcionado pela melhor compreensão da teoria. Tendem, por isso, a dar primado às técnicas, em detrimento do comportamento empático ou 
da percepção das emoções experienciadas pelos clientes, na medida em que estas são competências de difícil desenvolvimento, caso se recorra unicamente a uma abordagem teórica.

Como as emoções comunicam rapidamente ao meio envolvente as necessidades, os objectivos, e os estados emocionais da pessoa, essa informação irá regular o comportamento dos outros (Greenberg, 2002), o que aplicado à psicoterapia significa que as emoções expressas pelo cliente fornecem informação acerca das suas necessidades e objectivos, assim como acerca do modo como os comportamentos do terapeuta estão a ser recebidos e percepcionados.

A expressão facial apresenta então uma elevada importância no contexto terapêutico, nomeadamente porque olhar para uma face que expressa tristeza, que tem, por exemplo, as pupilas mais contraídas, vai fazer com que as pupilas do observador também se contraiam, o que tem efeitos neuronais ao nível da activação cerebral das regiões relacionadas com o processamento das emoções e com a empatia (Harrison, Singer, Rothstein, Dolan \& Critchley, 2006). Assim, o terapeuta conseguirá identificar a emoção expressa pelo cliente pela musculatura facial característica da emoção expressa, mas conseguirá empatizar também emocionalmente com ele, devido ao efeito fisiológico de activação emocional que se gera ao nível neuronal.

Assim, como o auto-relato relativamente a emoções com as quais os sujeitos estão em conflito se tem demonstrado pouco preciso, verifica-se ser vantajoso dar atenção à comunicação não verbal, sobre a qual os sujeitos exercem menor controlo, o que permite obter-se uma informação mais clara acerca da dimensão emocional (Machado, Beutler \& Greenberg, 1999). Passons (1975, citado por Cormier et al., 2009) confirma esta necessidade em terapia, referindo que os clientes estão mais conscientes das suas palavras do que dos seus comportamentos, sendo necessária da parte do terapeuta uma percepção da comunicação não verbal dos pacientes, seguida da sua exploração, para que eles obtenham uma melhor compreensão e consciência da mesma.

Além das descobertas supramencionadas acerca da influência da experiência no desenvolvimento destas capacidades relacionais, existem autores que referem que a capacidade de reconhecimento de emoções parece aumentar com a formação e treino específicos nessa área, nomeadamente Miguel e Noronha (2006), que verificaram que a capacidade de reconhecer a expressão 
das emoções, tanto em si mesmo como nos outros, é superior em estudantes de Psicologia, quando comparados com estudantes de Biologia e de Engenharia; e Machado e colaboradores (1999) que constataram que os terapeutas experientes identificam melhor as emoções do que estudantes universitários de Psicologia.

Machado e colaboradores (1999) observaram também que os terapeutas (indivíduos treinados) conseguem inferir o significado emocional na ausência de pistas contextuais, enquanto os indivíduos não treinados (estudantes) têm dificuldade, ou chegam mesmo a não conseguir fazê-lo, caso não tenham informação acerca do contexto do cliente. Importa salientar que a capacidade de reconhecimento de emoções tende a diminuir quando a informação é limitada a um único canal de comunicação (e.g. expressão facial, tom de voz) (Ekman, 1992), porém a capacidade de identificar a experiência emocional dos clientes apenas diminui nos estudantes, mantendo-se constante nos terapeutas, pelo que se depreende que enquanto estes fazem uma utilização correcta das pistas não verbais na identificação das emoções, os estudantes têm dificuldade em utilizálas quando são apresentadas sem a comunicação verbal, só conseguindo percebê-las se tiverem acesso em simultâneo às pistas verbais (Machado et al., 1999).

Através de uma integração da informação relativa a esta temática, revelase ambíguo relacionar a capacidade de percepção de emoções através da expressão facial com um dos tipos de empatia, pois embora o processamento da informação percepcionada visualmente inclua uma componente cognitiva, que recorre à memória emocional e atribui uma designação e significação à emoção expressa, existe igualmente sustentação teórica para o facto de a percepção das emoções gerar uma activação fisiológica mais automática no terapeuta, que experiencia a emoção percepcionada, podendo deste modo ressoar emocionalmente com o cliente. No entanto, há autores que referem que a empatia cognitiva não está relacionada com tarefas de reconhecimento de emoções (Besel \& Yuille, 2010), enquanto a empatia emocional está positivamente correlacionada com a capacidade de reconhecer adequadamente as expressões faciais das emoções (Riggio, Tucker \& Coffaro, 1989).

Ainda relativamente à empatia emocional, há inclusive autores que alertam para o facto de os indivíduos com resultados superiores na empatia emocional reagirem em concordância com a expressão facial que observam, o que não se verifica nos sujeitos que apresentam valores inferiores (Dimberg, Andréasson \& 
Thunberg, 2011). Este conjunto de evidências implica alguma independência entre os dois tipos de empatia, sustentada também pelo facto de os indivíduos com autismo apresentarem resultados baixos em compreensão empática (uma componente da empatia cognitiva), ainda que obtenham resultados elevados na empatia emocional (Smith, 2009).

Neste contexto, o presente estudo procura compreender a relação entre a empatia e a capacidade de reconhecimento de emoções, assim como perceber se estas competências apresentam diferenças entre terapeutas e estudantes de psicologia.

\section{MÉTODO}

\section{Participantes}

O presente estudo contou com a participação de estudantes dos cinco anos de formação do Mestrado Integrado em Psicologia, e de alguns psicoterapeutas. Trata-se de uma amostra objectiva, em que a escolha da população-alvo teve por base a intenção de uniformizar a qualidade da formação académica dos estudantes, pelo que foram estudadas as duas competências inter-pessoais em psicoterapeutas e em estudantes de diferentes anos da Faculdade de Psicologia da Universidade de Lisboa.

Colaboraram na investigação 113 sujeitos, dos quais 101 estudantes, com idades compreendidas entre os 18 e os 37 anos $(M=21,54$ e $D P=3,06)$, e 12 psicoterapeutas, pertencentes à faixa etária entre os 25 e os 52 anos $(M=35,42$ e $\mathrm{DP}=10,04)$, o que perfaz um total de 12 homens e 101 mulheres $(10,62 \%$ e $89,38 \%$ da amostra, respectivamente), uma proporção que reproduz de forma relativamente adequada a distribuição de géneros no curso de Psicologia desta faculdade.

Relativamente ao grau de formação, $10,62 \%$ da amostra são psicoterapeutas, $44,25 \%$ pertencem ao primeiro ciclo de estudos (dos quais $14,16 \%$ são do $1^{\circ}$ ano, $16,81 \%$ do $2^{\circ}$ ano e $13,28 \%$ do $3^{\circ}$ ano) e $45,13 \%$ são do $2^{\circ}$ ciclo de estudos $(30,08 \%$ de psicologia clínica e $15,05 \%$ de outras áreas de formação em psicologia). Os psicoterapeutas que se disponibilizaram para participar no estudo possuem credenciação em psicologia clínica, e uma formação pós-graduada em psicoterapia, ministrada pelas sociedades de psicoterapeutas ligadas às diversas correntes teóricas. 


\section{Instrumentos}

\section{Test de Empatía Cognitiva y Afectiva (TECA)}

Este instrumento foi desenvolvido por Bélén López-Péres, Irene FérnandezPinto e Francisco José Abad Garcia, e publicado por TEA Ediciones, em 2008, não tendo havido nenhuma adaptação do teste para Portugal até à actualidade. A primeira versão experimental portuguesa foi, assim, desenvolvida no presente estudo com base nas Directrizes de Adaptação de Testes Psicológicos (International Test Commission [ITC], 2010), sendo que a tradução realizada contou com a apreciação de três investigadores com experiência na adaptação de instrumentos de medida psicológica.

O TECA avalia a empatia e permite obter resultados em quatro subescalas diferentes: Adopção de Perspectiva, que se entende como capacidade de o indivíduo se colocar no lugar da outra pessoa e perspectivar a realidade sob o seu ponto de vista; Compreensão Emocional, que diz respeito à capacidade de reconhecer e compreender os estados emocionais e as intenções dos outros; Stress Empático, definido como a capacidade de partilhar as emoções negativas da outra pessoa, sintonizando-se emocionalmente com ela; e Alegria Empática, que é uma vertente positiva da escala anterior e diz respeito à capacidade de experienciar as emoções positivas da outra pessoa (López-Pérez, FérnandezPinto \& García, 2008). As duas primeiras subescalas (Adopção de Perspectiva e Compreensão Emocional), formam a escala de Empatia Cognitiva e, por sua vez, as duas últimas (Stress Empático e Alegria Empática) constituem a escala de Empatia Emocional.

No que diz respeito à aplicação do teste, a sua duração varia entre cinco a dez minutos e as respostas utilizam uma escala de Likert de cinco pontos, relativa à concordância com as afirmações: 1 - Totalmente em desacordo, 2 Parcialmente em desacordo, 3 - Neutro, 4 - Parcialmente de acordo, 5 Totalmente de acordo. Para a obtenção dos resultados das quatro subescalas, somam-se as pontuações correspondentes aos itens que as constituem, e, no final, os somatórios são uma vez mais adicionados, de forma a permitir a obtenção dos totais de cada indivíduo para a Empatia Cognitiva, para a Empatia Emocional e para a Escala Completa.

No que diz respeito à consistência interna da versão original do teste, a escala completa apresenta um alfa de Cronbach de 0,86. Nas subescalas do 
instrumento, os coeficientes são de 0,70 para a Adopção de Perspectiva, de 0,74 para a Compreensão Emocional, de 0,78 para o Stress Empático e de 0,75 para a Alegria Empática.

Teste de Percepção de Emoções Primárias (PEP)

O instrumento foi desenvolvido na Universidade de São Francisco, em São Paulo (Brasil), por Miguel e Primi, em 2010, e consiste na apresentação de vídeos de curta duração, onde um indivíduo, masculino ou feminino, expressa uma determinada emoção básica, e em que se solicita que a pessoa identifique qual a emoção ou emoções expressas e diga se é/são emoções genuínas/verdadeiras ou falsas/dissimuladas. As emoções primárias utilizadas no teste têm por base a teoria psicoevolucionista de Plutchik (2000), que contempla oito emoções: alegria, aceitação (que no PEP foi designada por "amor"), medo, surpresa, nojo, raiva e expectativa (identificada no PEP como "curiosidade").

Os sujeitos que foram filmados estavam em frente a um computador, onde visualizavam imagens de várias temáticas, tais como faces, paisagens, objectos, eventos e fenómenos naturais, passíveis de gerar uma emoção no observador. Foram também utilizados trechos de filmes para desencadear ansiedade e medo, pois imagens estáticas poderiam não possuir a intensidade pretendida. Caso se pretendesse que o sujeito dissimulasse a emoção, era apresentada uma informação prévia no ecrã do computador, relativamente à emoção que teria de ser expressa, independentemente do que fosse visto de seguida.

A aplicação tem duração média de trinta minutos e, para cada item apresentado, o sujeito pode acumular até um total de oito pontos, sendo que recebe um ponto pela selecção da emoção correcta e um ponto por cada emoção acertadamente não seleccionada, isto é, caso a emoção expressa seja alegria, será atribuído um ponto à escolha da emoção alegria e um ponto por cada uma das outras sete emoções que não se encontram expressas no vídeo, e que o sujeito correctamente não seleccionou.

Os somatórios da identificação do carácter verdadeiro ou falseado das emoções são independentes da identificação das emoções propriamente ditas, sendo que os sujeitos recebem um ponto sempre que acertam no que diz respeito à veracidade ou falsidade da emoção expressa em cada item. Esta componente do teste não foi utilizada na presente investigação, por se ter 
optado por circunscrever as variáveis em estudo à capacidade de identificação das emoções expressas pelos outros, independentemente da sua autenticidade.

Por fim, relativamente à consistência do instrumento original, importa salientar que apresentou um bom coeficiente de consistência interna, $a=0,81$, relativamente à escala global de identificação de emoções expressas facialmente. Contudo, os autores do teste identificarm a existência de cinco factores explicativos da variância, o que aparentemente compromete a unidimensionalidade das medidas proporcionadas pelo instrumento, por não existir um factor explicativo primordial (Miguel \& Primi, 2010), sendo que, neste sentido, o resultado global poderá constituir uma medida pouco legítima, eventualmente desprovida de significado psicológico.

\section{Procedimentos}

As aplicações decorreram em salas de computadores da Faculdade de Psicologia da Universidade de Lisboa, na medida em que os sujeitos responderam a ambos os instrumentos em suporte informatizado, com o sistema operativo Windows XP (por imposição das configurações do PEP). Sempre que possível, realizou-se a recolha dos dados num ambiente estandardizado, sem elementos distractores e, embora tenham decorrido várias aplicações em simultâneo, a explicação relativa aos passos a realizar foi sempre facultada individualmente.

Antes da sessão de avaliação, os participantes eram relembrados do tempo de duração da aplicação (comunicado aquando do pedido de colaboração) e informados de que iriam preencher alguns dados pessoais (habilitações académicas, idade e género), que seriam seguidas de um questionário de autorelato acerca das relações interpessoais e de uma tarefa de visionamento de um conjunto de vídeos, para identificação de emoções expressas facialmente pelos indivíduos.

Todos os sujeitos foram informados de que Ihes seria atribuído um código de identificação, correspondente ao número de aplicações realizadas até ao momento, de modo a salvaguardar a confidencialidade das respostas dadas, mas permitindo que a informação obtida em ambos os testes aplicados pudesse ser correlacionada. No final, foi-Ihes solicitado que se pronunciassem criticamente ou que fizessem sugestões que julgassem pertinentes e relevantes, e procedeu-se à 
explicação do propósito da investigação, frisando a disponibilidade posterior dos resultados.

\section{RESULTADOS}

\section{Estatísticas Descritivas}

Na Tabela 1 encontram-se as estatísticas descritivas (medianas, médias, desvios-padrão, mínimos e máximos) para cada grupo, relativamente aos somatórios globais de ambos os instrumentos utilizados no presente estudo. Por sua vez, na Tabela 2 encontram-se as mesmas informações referentes às subamostras que serão comparadas em função das hipóteses.

Tabela 1.

Estatísticas descritivas dos somatórios do PEP e do TECA.

\begin{tabular}{|c|c|c|c|c|c|c|c|c|c|c|}
\hline \multirow{2}{*}{$\begin{array}{c}\text { Grupos } \\
\text { (n) }\end{array}$} & \multicolumn{5}{|c|}{ PEP } & \multicolumn{5}{|c|}{ TECA } \\
\hline & $M$ & $\mathrm{~m}$ & $\mathrm{dp}$ & Mín & Máx & $M$ & $\mathrm{~m}$ & $\mathrm{dp}$ & Mín & Máx \\
\hline 10 Ano (16) & 221,25 & 222 & 4,39 & 210 & 228 & 123,44 & 123 & 6,69 & 108 & 133 \\
\hline $2^{\circ}$ Ano (19) & 221,42 & 223 & 5,37 & 209 & 230 & 128,05 & 131 & 8,82 & 114 & 142 \\
\hline $3^{\circ}$ Ano (15) & 222,27 & 221 & 3,99 & 216 & 228 & 129,47 & 128 & 11,78 & 108 & 150 \\
\hline $4^{\circ}$ Clínica (15) & 218,73 & 221 & 6,19 & 207 & 230 & 123,00 & 123 & 10,25 & 98 & 137 \\
\hline $4^{\circ} \mathrm{N}$. Clínica (6) & 219,67 & 220 & 6,38 & 209 & 229 & 119,17 & 122 & 8,45 & 104 & 126 \\
\hline $5^{\circ}$ Clínica (19) & 221,21 & 222 & 4,44 & 213 & 228 & 128,95 & 131 & 10,77 & 104 & 147 \\
\hline $5^{\circ} \mathrm{N}$. Clínica (11) & 223,55 & 224 & 5,89 & 213 & 232 & 128,82 & 128 & 9,76 & 115 & 171 \\
\hline Terapeutas (12) & 222,75 & 222 & 3,42 & 218 & 228 & 130,83 & 130 & 7,18 & 120 & 150 \\
\hline
\end{tabular}

Nota: (N. Clínica) - Não Clínica

\section{Consistência Interna}

A consistência interna do PEP, avaliada através do cálculo do alfa de Cronbach, aplicado à escala global de identificação das emoções expressas, foi de $a=0,32$. No que diz respeito ao TECA, a precisão da versão traduzida na presente investigação foi calculada para a escala completa do instrumento, $a=0,77$, assim como para as várias subescalas que o constituem: a Adopção de Perspectiva $(a=0,79)$, a Compreensão Emocional $(a=0,76)$, o Stress Empático $(a=0,73)$ e a Alegria Empática $(a=0,60)$. Verificou-se interessante e pertinente avaliar a consistência também das duas escalas compósitas, a Empatia Cognitiva $(a=0,82)$ e a Empatia Emocional $(a=0,72)$, ainda que essa informação não seja apresentada na versão original do teste. 
Tabela 2.

Estatísticas descritivas dos somatórios do PEP e do TECA (Subamostras).

\begin{tabular}{|c|c|c|c|c|c|c|c|c|c|c|}
\hline \multirow{2}{*}{$\begin{array}{c}\text { Grupos } \\
\text { (n) }\end{array}$} & \multicolumn{5}{|c|}{ PEP } & \multicolumn{5}{|c|}{ TECA } \\
\hline & $M$ & $\mathrm{~m}$ & $\mathrm{dp}$ & Mín & Máx & M & $\mathrm{m}$ & $\mathrm{dp}$ & Mín & Máx \\
\hline Estudantes (101) & 221,21 & 222 & 5,15 & 207 & 232 & 126,50 & 127 & 9,94 & 98 & 151 \\
\hline 10 Ciclo (50) & 221,62 & 222 & 4,61 & 209 & 230 & 127,00 & 127 & 9,40 & 108 & 150 \\
\hline $1^{\circ}$ C. e $4^{\circ}$ A. (71) & 220,85 & 221 & 5,19 & 207 & 230 & 125,49 & 126 & 9,71 & 98 & 150 \\
\hline 20 Ciclo (51) & 220,80 & 221 & 5,66 & 207 & 232 & 126,02 & 126 & 10,52 & 98 & 151 \\
\hline Clínica (45) & 220,96 & 221 & 5,62 & 207 & 232 & 126,93 & 128 & 10,51 & 98 & 151 \\
\hline N. Clínica (6) & 219,67 & 220 & 6,38 & 209 & 229 & 119,17 & 122 & 8,45 & 104 & 126 \\
\hline 50 Clínica (19) & 221,21 & 222 & 4,44 & 213 & 228 & 128,95 & 131 & 10,77 & 104 & 147 \\
\hline Terapeutas (12) & 222,75 & 222 & 3,42 & 218 & 228 & 130,83 & 130 & 7,18 & 120 & 150 \\
\hline
\end{tabular}

Nota: (10 C. e 40 A.) - $1^{\circ}$ Ciclo e 40 Ano; (N. Clínica) - Não Clínica

\section{Correlações}

As correlações entre as variáveis consideradas, calculadas através do Coeficiente de Spearman, são apresentadas na Tabela 3, sendo necessário ressalvar que no TECA as correlações entre as partes e o todo (que se encontram sombreadas na tabela) são espúrias. A partir da análise dos resultados obtidos, é possível verificar que o PEP apresenta uma correlação positiva com a Empatia, contudo o coeficiente não atinge o critério de significância estatística.

Nas correlações espúrias é possível verificar que, tal como seria esperado, a Empatia Cognitiva (E_COG) se encontra significativamente correlacionada com a Adopção de Perspectiva (AP) e a Compreensão Emocional (CE), e por sua vez a Empatia Emocional (E_EMO) com o Stress Empático (SE) e a Alegria Empática (AE). Relativamente ao Somatório do TECA, observa-se a existência de uma correlação mais elevada com a Empatia Cognitiva, não obstante a relação positiva e significativa que apresenta com a Empatia Emocional.

Por fim é ainda possível verificar que a Empatia Cognitiva apresenta correlações significativas com as escalas de Empatia Emocional, a AE e o SE, sendo contudo a relação com esta última negativa, pelo que os sujeitos que apresentem um resultado superior nesta subescala, tenderão a manifestar maior Empatia Emocional e menor Empatia Cognitiva. No que diz respeito às duas dimensões da empatia, o facto de apresentarem uma correlação 
consideravelmente baixa e negativa, aponta no sentido da independência entre as duas componentes, sustentada pela literatura.

Tabela 3.

Correlações ordinais entre as variáveis em estudo $(N=113)$.

\begin{tabular}{cccccccc}
\hline & AP & CE & SE & AE & E_COG & E_EMO & TECA \\
\hline CE & $0,39 * *$ & & & & & & \\
SE & $-0,17$ & $-0,19^{*}$ & & & & & \\
AE & 0,17 & $0,22^{*}$ & $0,21^{*}$ & & & & \\
E_COG & $0,79 * *$ & $0,85^{* *}$ & $-0,21^{*}$ & $0,27^{* *}$ & & & \\
E_EMO & $-0,03$ & $-0,08$ & $0,90^{* *}$ & $0,57^{* *}$ & $-0,05$ & & \\
TECA & $0,58^{* *}$ & $0,63^{* *}$ & $0,41^{* *}$ & $0,57^{* *}$ & $0,74 * *$ & $0,58 * *$ & \\
PEP & 0,12 & 0,06 & 0,09 & 0,15 & 0,08 & 0,16 & 0,17 \\
\hline
\end{tabular}

Nota: AP (Adopção de Perspectiva), CE (Compreensão Emocional), SE (Stress Empático), AE (Alegria Empática), E_COG (Empatia Cognitiva), E_EMO (Empatia Emocional), TECA/PEP (Somatórios totais)

$* p<0,05 * * p<0,01$

\section{Análise Multivariada Não Paramétrica}

A partir das comparações realizadas entre os diferentes grupos, em função das duas variáveis dependentes, os Somatórios do PEP e do TECA, verifica-se que não são significativas nesta amostra as diferenças entre os psicoterapeutas e os estudantes $(p=0,31)$, entre os estudantes do $5^{\circ}$ ano de Clínica e os estudantes de anos inferiores $(p=0,18)$, entre os estudantes de 10 Ciclo e os de 20 Ciclo $(p=0,74)$ e entre os estudantes de Psicologia Clínica e os de outras áreas de formação do Mestrado Integrado em Psicologia $(p=0,16)$.

Por sua vez, a análise relativa aos dois tipos de empatia identificou diferenças significativas em alguns grupos, relativamente à Empatia Cognitiva $(p=0,00)$. Na Tabela 4 são apresentados os índices relativos às diferenças entre os grupos comparados.

Tendo por base as diferenças significativas entre os grupos, relativamente à Empatia Cognitiva, considerou-se pertinente proceder à comparação dos grupos, em função das quatro subescalas constituintes do TECA. Os resultados indicaram que entre os psicoterapeutas $(m=83,38)$ e os estudantes $(m=53,87)$ existem diferenças significativas na Compreensão Emocional $(p=0,00)$; que os estudantes do $5^{\circ}$ ano de Clínica $(m=58,32)$ diferem dos estudantes pertencentes aos restantes anos anteriores $(m=42,07)$, no que diz respeito à Alegria Empática $(p=0,02)$; e que os estudantes de Mestrado em Clínica $(m=27,80)$ apresentam diferenças ao nível da Compreensão Emocional $(p=0,02)$ quando comparados 
com os que Não são de Clínica $(m=12,50)$. Obteve-se também a informação de que não existem diferenças significativas nesta amostra entre o $1^{\circ}$ e o $2^{\circ}$ Ciclo do Mestrado Integrado em Psicologia $(p=0,21)$.

Tabela 4.

Diferenças entre os grupos, em função da Empatia Cognitiva.

\begin{tabular}{|c|c|c|c|c|c|c|c|}
\hline & $1^{\circ} \mathrm{Ano}$ & $2^{\circ}$ Ano & $3^{\circ}$ Ano & $4^{0}$ Clínica & $\begin{array}{l}4^{0} \text { Não } \\
\text { Clínica }\end{array}$ & $5^{\circ}$ Clínica & $\begin{array}{l}5^{0} \text { Não } \\
\text { Clínica }\end{array}$ \\
\hline $2^{\circ}$ Ano & $-7,39$ & & & & & & \\
\hline $3^{\circ}$ Ano & $-30,31 *$ & $-22,92 *$ & & & & & \\
\hline $4^{0}$ Clínica & $-9,81$ & $-2,42$ & 20,50 & & & & \\
\hline $4^{0}$ Não Clínica & 13,19 & 20,58 & $43,50 *$ & 23,00 & & & \\
\hline $5^{\circ}$ Clínica & $-26,71 *$ & $-19,32$ & 3,61 & $-16,89$ & $-39,89 *$ & & \\
\hline $5^{\circ}$ Não Clínica & $-16,45$ & $-9,06$ & 13,86 & $-6,64$ & $-29,64$ & 10,26 & \\
\hline Terapeutas & $-40,98 *$ & $-33,59 *$ & $-10,67$ & $-31,17 *$ & $-54,17 *$ & $-14,27$ & $-24,53$ \\
\hline
\end{tabular}

\section{DISCUSSÃO DOS RESULTADOS}

Nos resultados correlacionais foi possível verificar que o Stress Empático se relaciona negativa e significativamente com a Empatia Cognitiva, o que pode ser justificado a partir das características da amostra, na medida em que esta é constituída unicamente por estudantes de Psicologia e psicoterapeutas, que ao longo da sua formação académica apreendem que, numa relação de ajuda, deve existir empatia para com as emoções negativas expressas pelo outro, devolvendo-lhe um sentimento de compreensão e de adequação emocional, sendo contudo importante manter a distância emocional necessária para não perder a objectividade (Gladstein, 1983). Hall, Davis e Connelly (2000) salientam, inclusive, que os melhores terapeutas experimentam um envolvimento com compreensão empática do paciente, embora salvaguardem que não apresentam um envolvimento negativo que poderia despertar angústia pessoal.

Ainda acerca deste instrumento, é de notar alguma independência relativa dos dois tipos de empatia, na medida em que apresentam uma correlação próxima de zero. Os resultados confirmam, desta forma, a teoria antes referida, relativa ao desequilíbrio numa mesma pessoa entre os dois tipos de empatia, e que em casos extremos (como o autismo) se traduz num funcionamento social menos adequado. Contudo, os resultados requerem interpretação cautelosa, pois 
os instrumentos de auto-relato, ao envolverem apenas auto-relato, poderão produzir resultados enviesados por uma percepção individual distorcida.

No que diz respeito à relação entre ambos os instrumentos, foi possível constatar que a correlação entre o PEP e o TECA não foi significativa, o que pode decorrer de factores distintos mas complementares, entre os quais se destaca a baixa consistência interna e a baixa variabilidade do primeiro, e a baixa covariância existente entre ambos em resultado da dissemelhança de método empregue na medição. Os baixos níveis de consistência interna do PEP traduzem-se numa menor proporção de variância verdadeira (há muito ruído, ou factores de erro aleatório que pesam nas diferenças individuais medidas), ao que acresce a restrição de amplitude dos resultados (patente nas estatísticas descritivas, Tabela 1), o que implica menores correlações com outras variáveis.

A baixa covariância entre os dois testes pode, por outro lado, decorrer da dissemelhança de métodos de medida utilizados: enquanto no TECA se recorre ao auto-relato para estimar a competência empática dos sujeitos (prova de comportamento típico com respostas numa escala de Likert), a capacidade de Percepção de Emoções Primárias é avaliada no PEP de acordo com o desempenho numa tarefa de identificação de emoções em expressões faciais (tarefa de desempenho máximo), com respostas certas e erradas. A percentagem de variância partilhada pelas variáveis será necessariamente mais reduzida, em consequência da dissemelhança de método, e apenas traduzirá, possivelmente, a natureza próxima dos construtos medidos. Assim, a correlação positiva é, neste contexto, expressiva e pode sugerir, como previsto, a existência de uma correlação positiva entre a empatia e a percepção de emoções.

Verifica-se também que a baixa consistência interna do PEP para a amostra em questão contrasta de algum modo com os elevados índices de precisão obtidos na população brasileira, onde foi construído o teste. Importa, contudo, reconhecer que as características de elevada homogeneidade etária e de género da amostra, mais ainda, composta por pessoas de uma única instituição, profissionais e estudantes de Psicologia, tornam pouco legítima a comparação com o estudo metrológico brasileiro, que englobou não só sujeitos com frequência universitária em Psicologia, mas também em Administração, tendo contado inclusive com a participação de indivíduos que não frequentavam o ensino superior. Ainda assim, esta constatação faz emergir a necessidade de aprofundar a consideração da temática da equivalência intercultural no que se 
refere ao reconhecimento das emoções básicas, no sentido de perceber em que medida as diferenças se podem atribuir a características culturais não universais.

No que diz respeito à reduzida variabilidade do teste, esta pode ter a sua origem em problemas de equivalência inter-cultural, ou na ausência de unidimensionalidade na medição do construto. Miguel e Primi (2010) referem que os itens do PEP parecem agrupar-se em função da emoção que representam, o que poderia indicar a existência de diferenças no processamento das várias emoções; contudo, o padrão não é totalmente claro, existindo emoções sem saturação apreciável nos factores identificados.

Ainda acerca deste instrumento, seria esperado que apresentasse uma correlação positiva com a Empatia Emocional, na medida em que os indivíduos que apresentam valores mais elevados nesta capacidade empática, reproduzem e identificam a expressão facial observada de um modo automático (SonnbyBorgström, 2002). Esta relação não se verificou, no entanto, assim como também não foram encontradas diferenças significativas entre os diferentes grupos na percepção de emoções primárias, o que pode dever-se aos factores aludidos anteriormente (baixa variabilidade e precisão do PEP).

Por sua vez, no que diz respeito ao TECA, não foram igualmente encontradas diferenças significativas entre nenhum dos grupos, excepto na análise das subescalas constituintes do teste, pois a empatia cognitiva apresentou diferenças entre os grupos, sendo superior nos indivíduos que possuem maior formação e experiência (estudantes do $5^{\circ}$ ano de clínica e psicoterapeutas), apesar de não se verificar um aumento gradual em função da progressão na formação. Estes resultados sugerem que a compreensão dos estados emocionais dos outros e das situações que se encontram na sua origem poderá estar a ser desenvolvida, na formação académica especializada em Psicologia Clínica.

\section{CONSIDERAÇÕES FINAIS}

Sabe-se que todos os indivíduos possuem os músculos faciais necessários (músculos básicos) para produzir os movimentos fundamentais à expressão facial, que é então universal (Waller, Cray \& Burrows, 2008). Contudo, pessoas de diferentes culturas recorrem a diferentes estratégias perceptivas para processar a face humana, pelo que, dependendo da cultura a que pertencem, os sujeitos podem centrar a sua atenção nos olhos, ou por exemplo no centro da 
face, sendo este um factor que pode influenciar a informação recebida (Blais, Jack, Scheepers, Fiset \& Caldara, 2008). Assim, podem existir diferenças culturais entre Portugal e o Brasil, no que diz respeito à apreciação perceptiva, não estando em causa a universalidade da configuração da expressão facial, mas sim a percepção e interpretação da informação observada. No entanto, esta justificação carece de sustentação holística, na medida em que actualmente existe uma elevada diversidade cultural na população portuguesa, que dota naturalmente os indivíduos da capacidade de ultrapassar estas diferenças, para haver entendimento mútuo na interacção social.

Neste sentido, esta questão sugere uma outra consideração relativa à componente semântica da tarefa do PEP, na medida em que é solicitado aos sujeitos que escolham denominações de emoções para definir a expressão facial que estão a observar. Fernández-Dols, Carrera, Barchard e Gacitua (2008) referem que o reconhecimento de emoções através das expressões faciais não é um processo de fácil categorização automática, mas sim um processo inferencial, tanto da emoção presente na expressão facial, como da informação semântica que Ihe está associada. Neste sentido, há então a possibilidade de ocorrer uma dissociação entre o mecanismo visual, responsável pela descodificação da emoção expressa na face, e um outro mecanismo, de nível superior de processamento, que associa a representação facial observada à informação semântica disponível, gerando a identificação de uma denominação para a emoção expressa (Balconi \& Lucchiari, 2007).

Já no que diz respeito aos resultados do TECA, importa salientar que, apesar de a empatia cognitiva se manifestar como superior nos estudantes do $5^{\circ}$ ano de Clínica e nos psicoterapeutas, também o $3^{\circ}$ ano do $1^{\circ}$ Ciclo de estudos em Psicologia apresenta valores elevados. Os valores superiores dos três grupos proeminentes poderão resultar de um desenvolvimento desta capacidade, em função da experiência quotidiana, não havendo influência directa da formação em Psicologia nos resultados. Porém, apesar de as pessoas que se tornam terapeutas levarem consigo já atributos adquiridos ao longo do seu desenvolvimento e interacções sociais, as quais influenciam as suas competências empáticas (Machado et al., 1999), ao longo do tempo vão adquirindo um conhecimento empático superior, que inicialmente é declarativo (importância da empatia) e passa a ser posteriormente processual (modo como 
agir para ser empático), como resultado do treino prático ao nível das suas atitudes e competências (Thwaites \& Bennett-Levy, 2007).

\section{REFERÊNCIAS}

Balconi, M., \& Lucchiari, C. (2007). Consciousness and emotional facial expression recognition: Subliminal/supraliminal stimulation effect on $\mathrm{n} 200$ and p300 ERPs. Journal of Psychophysiology, 21(2), 100-108. doi:10.1027/0269-8803.21.2.100

Bennett-Levy, J., \& Thwaites, R. (2009). Self and self reflection in the therapeutic relationship: A conceptual map and practical strategies for the training supervision and self-supervision of interpersonal skills. In P. Gilbert \& R. Leahy (Eds.), The Therapeutic Relationship in the Cognitive Behavioral Psychotherapies (pp. 255-281). New York: Routledge.

Besel, L. D. S., \& Yuille, J. C. (2010). Individual differences in empathy: The role of facial expression recognition. Personality and Individual Differences, 49(2), 107-112. doi:10.1016/j.paid.2010.03.013

Blais, C., Jack, R. E., Scheepers, C., Fiset, D., \& Caldara, R. (2008). Culture shapes how we look at faces. PLOS ONE, 3(8), 1-8. doi: $10.1371 /$ journal.pone.0003022

Bohart, A. C., \& Greenberg, L. S. (1997). Empathy: Where are we and where do we go from here? In A. C. Bohart, L. S. Greenberg, A. C. Bohart, L. S. Greenberg (Eds.), Empathy reconsidered: New directions in psychotherapy (pp. 419-449). American Psychological Association. doi:10.1037/10226-031

Bordin, E. S. (1979). The generalizability of the psychoanalytic concept of the working alliance. Psychotherapy: Theory, Research \& Practice, 16(3), 252260. doi: $10.1037 / \mathrm{h} 0085885$

Cormier, S., Nurius, P., \& Osborn, C. (2009). Interviewing and change strategies for helpers: Fundamental skills and cognitive behavioral interventions. Australia: Thompson Brooks.

Dimberg, U., Andréasson, P., \& Thunberg, M. (2011). Emotional empathy and facial reactions to facial expressions. Journal of Psychophysiology, 25(1), 26-31. doi: 10.1027/0269-8803/a000029

Ekman, P. (1992). Facial expressions of emotion: New findings, new questions. Psychological Science, 3(1), 34-38. 
Fernández-Dols, J., Carrera, P., Barchard, K. A., \& Gacitua, M. (2008). False recognition of facial expressions of emotion: Causes and implications. Emotion, 8(4), 530-539. doi:10.1037/a0012724

Fernández-Pinto, I., López-Pérez, B., \& Márquez, M. (2008). Empatía: Medidas, teorias y aplicaciones en revisión. Anales de Psicología, 24(2), 284-298.

Gilbert, P. (2009). Evolved minds and compassion in the therapeutic relationship. In P. Gilbert \& R. Leahy (Eds.), The therapeutic relationship in the cognitive behavioral psychotherapies (pp. 106-142). New York: Routledge.

Gilbert, P., \& Leahy, R. (2009). Introduction and overview: Basic issues in the therapeutic relationship. In P. Gilbert \& R. Leahy (Eds.), The therapeutic relationship in the cognitive behavioral psychotherapies (pp. 3-23). New York: Routledge.

Gladstein, G. A. (1983). Understanding empathy: Integrating counseling, developmental, and social psychology perspectives. Journal of Counseling Psychology, 30(4), 467-482. doi:10.1037/0022-0167.30.4.467

Greenberg, L. S. (2002). Emotions and emotional intelligence. In L. S. Greenberg (Ed.), Emotion-focused therapy: Coaching clients to work through their feelings (pp. 3-38). American Psychological Association. doi: $10.1037 / 10447-001$

Greenberg, L. S. (2009). Emotion in the therapeutic relationship in emotionfocused therapy. In P. Gilbert \& R. Leahy (Eds.), The therapeutic relationship in the cognitive behavioral psychotherapies (pp. 43-62). New York: Routledge.

Hall, J. A., Davis, M. H., \& Connelly, M. (2000). Dispositional empathy in scientists and practitioner psychologists: Group differences and relationship to self-reported professional effectiveness. Psychotherapy: Theory, Research, Practice, Training, 37(1), 45-56. doi:10.1037/h0087758

Harrison, N. A., Singer, T., Rotshtein, P., Dolan, R. J., \& Critchley, H. D. (2006). Pupillary contagion: Central mechanisms engaged in sadness processing. Social Cognitive \& Affective Neuroscience, 1(1), 5-17. doi: 10.1093/scan/nsl006

International Test Commission (2010). International Test Commission guidelines for translating and adapting tests. Retirado de http://www.intestcom.org López-Pérez, B., Férnandez-Pinto, I., García, F.J.A. (2008). TECA: Test de Empatía Cognitiva y Afectiva (Manual). Madrid: TEA Ediciones, S.A. 
Machado, P. P. P., Beutler, L. E., \& Greenberg, L. S. (1999). Emotion recognition in psychotherapy: Impact of therapist level of experience and emotional awareness. Journal of Clinical Psychology, 55(1), 39-57.

Mayer, J. D., Salovey, P., \& Caruso, D. R. (2004). Emotional intelligence: Theory, findings, and implications. Psychological Inquiry, 15(3), 197-215.

Miguel, F. K., \& Noronha, A. P. P. (2006). Estudo da inteligência emocional em cursos universitários. In A. P. Soares, S. Araújo \& S. Caires (Eds.). Avaliação psicológica e contextos (pp. 613-619). Braga, Portugal: Psiquilíbrios.

Miguel, F. K. \& Primi, R. (2010). Teste Informatizado de Percepção de Emoções Primárias para avaliação de adultos. In M. C. R. A. Joly \& C. T. Reppold (Eds.), Estudos de testes informatizados para avaliação psicológica (pp. 231-245). São Paulo: Casa do Psicólogo.

Plutchik, R. (2000). A psychoevolutionary theory of emotion. In R. Plutchik (Ed.), Emotions in the practice of psychotherapy: Clinical implications of affect theories (pp. 59-79). American Psychological Association.

Riggio, R. E., Tucker, J. \& Coffaro, D. (1989). Social skills and empathy. Personality and Individual Differences, 10(1), 93-99.

Rogers, C. R. (1974). A terapia centrada no paciente (M. C. Ferreira, Trad.). Lisboa: Moaes Editores. (Obra original publicada em 1951)

Smith, A. (2009). The empathy imbalance hypothesis of autism: A theoretical approach to cognitive and emotional empathy in autistic development. The Psychological Record, 59(3), 489-510.

Sonnby-Borgström, M. (2002). Automatic mimicry reactions as related to differences in emotional empathy. Scandinavian Journal of Psychology, 43(5), 433-443. doi:10.1111/1467-9450.00312

Thwaites, R., \& Bennett-Levy, J. (2007). Conceptualizing empathy in cognitive behaviour therapy: Making the implicit explicit. Behavioural and Cognitive Psychotherapy, 35(5), 591-612. doi:10.1017/S1352465807003785

Vasco, A. B. (2007). Quando menos é melhor: A arte de comunicar em psicoterapia. Comunicação no Encontro Nacional "Comunicação em Saúde", Universidade dos Açores, Ponta Delgada.

Vasco, A. B. (2005). Creio num engenho mais fecundo de harmonizar as partes dissonantes: Fundamentação para a integração em psicoterapia. PsiLogos, $1(2), 77-94$. 
Waller, B. M., Cray, J. R., \& Burrows, A. M. (2008). Selection for universal facial emotion. Emotion, 8(3), 435-439. doi:10.1037/1528-3542.8.3.435

Contato: ritapalhoco@gmail.com, mjafonso@fp.ul.pt

Recebido em: 21/12/2011

Revisado em: 28/12/2011

Aceito em: 30/12/2011 